Note: The following material may be protected by copyright law (Title 17, U.S. Code)

\title{
Event-Free Survival, a Prostate-Specific Antigen-Based Composite End Point, Is Not a Surrogate for Overall Survival in Men With Localized Prostate Cancer Treated With Radiation
}

Wanling Xie, MS ${ }^{1}$; Meredith M. Regan, ScD ${ }^{1}$; Marc Buyse, ScD²; Susan Halabi, PhD ${ }^{3}$; Philip W. Kantoff, MD ${ }^{4}$ Oliver Sartor, MD ${ }^{5}$; Howard Soule, PhD ; Donald Berry, PhD7 Noel Clarke, MBBS ${ }^{8}$; Laurence Collette, PhD ${ }^{9}$; Anthony D'Amico, MD, PhD ${ }^{10}$; Richard De Abreu Lourenco, PhD ${ }^{11}$; James Dignam, PhD ${ }^{12}$; Mario Eisenberger, MD ${ }^{13}$; Nicholas James, MBBS, PhD ${ }^{14}$; Karim Fizazi, MD, PhD ${ }^{15}$; Silke Gillessen, MD ${ }^{16}$; Yohann Loriot, MD, PhD ${ }^{15}$; Nicolas Mottet, MD, PhD ${ }^{17}$; Wendy Parulekar, MD ${ }^{18}$; Howard Sandler, MD ${ }^{19}$; Daniel E. Spratt, MD ${ }^{20}$; Matthew R. Sydes, MSc ${ }^{21}$; Bertrand Tombal, MD, PhD ${ }^{22}$; Scott Williams, MBBS, MD ${ }^{23}$; and Christopher J. Sweeney, MBBS ${ }^{24}$ on behalf of the ICECaP Working Group

ASSOCIATED CONTENT

Appendix

Data Supplement

Author affiliations and support information (if applicable) appear at the end of this article.

Accepted on April 24, 2020 and published at ascopubs.org/journal/ jco on June 18, 2020: DOl https://doi.org/10. 1200/JC0.19.03114

The pharmaceutical companies who provided financial support as investigator-initiated grants had no input into the design or interpretation of the results.

PURPOSE Recently, we have shown that metastasis-free survival is a strong surrogate for overall survival (OS) in men with intermediate- and high-risk localized prostate cancer and can accelerate the evaluation of new (neo) adjuvant therapies. Event-free survival (EFS), an earlier prostate-specific antigen (PSA)-based composite end point, may further expedite trial completion.

METHODS EFS was defined as the time from random assignment to the date of first evidence of disease recurrence, including biochemical failure, local or regional recurrence, distant metastasis, or death from any cause, or was censored at the date of last PSA assessment. Individual patient data from trials within the Intermediate Clinical Endpoints in Cancer of the Prostate-ICECaP-database with evaluable PSA and disease follow-up data were analyzed. We evaluated the surrogacy of EFS for OS using a 2-stage meta-analytic validation model by determining the correlation of EFS with OS (patient level) and the correlation of treatment effects (hazard ratios [HRs]) on both EFS and OS (trial level). A clinically relevant surrogacy was defined a priori as an $R^{2} \geq 0.7$.

RESULTS Data for 10,350 patients were analyzed from 15 radiation therapy-based trials enrolled from 1987 to 2011 with a median follow-up of 10 years. At the patient level, the correlation of EFS with OS was $0.43(95 \% \mathrm{Cl}$, 0.42 to 0.44$)$ as measured by Kendall's tau from a copula model. At the trial level, the $R^{2}$ was $0.35(95 \% \mathrm{Cl}, 0.01$ to 0.60 ) from the weighted linear regression of $\log (\mathrm{HR})-\mathrm{OS}$ on $\log (\mathrm{HR})$-EFS.

CONCLUSION EFS is a weak surrogate for OS and is not suitable for use as an intermediate clinical end point to substitute for OS to accelerate phase III (neo)adjuvant trials of prostate cancer therapies for primary radiation therapy-based trials.

J Clin Oncol 38:3032-3041. @ 2020 by American Society of Clinical Oncology

\section{INTRODUCTION}

Despite various strategies for the early detection of prostate cancer, more than one half of all deaths occur in men who initially presented with localized disease. ${ }^{1}$ Curing metastatic prostate cancer is still a formidable challenge, and the most likely near-term strategy to substantially decrease the approximately 300,000 prostate cancer deaths that occur worldwide from the 1.1 million newly diagnosed cases is by preventing relapses from intermediate- and high-risk localized disease with more effective (neo)adjuvant systemic therapy. ${ }^{2-5}$
Improvements in systemic therapies increase the longevity of some men with metastatic hormonesensitive prostate cancer and castration-resistant prostate cancer (CRPC) ${ }^{6-18}$ When metastases are occult to conventional computerized tomography and technetium bone-scan imaging, they are more sensitive to testosterone-suppression therapy, resulting in an increase in the chance of cure when systemic therapies are given with local therapy. ${ }^{5,19,20}$ Potential eradication of micrometastases with new therapies may further decrease the number of men who die as a result of prostate cancer. When the 


\section{CONTEXT}

\section{Key Objectives}

To determine whether event-free survival (EFS), an early prostate-specific antigen (PSA)-based composite end point can be used as an intermediate clinical end point and serve as a surrogate for overall survival (OS) in localized prostate cancer. If surrogacy was established, the secondary objective was to detail how to deploy EFS as an end point to expedite completion of randomized phase III clinic trials in this setting.

\section{Knowledge Generated}

EFS has very a low correlation with OS in men with localized prostate cancer treated with radiation, and is therefore a weak surrogate for OS.

\section{Relevance}

Unlike metastasis-free survival, EFS is not suitable for use as an intermediate clinical end point to substitute for OS to accelerate phase III (neo)adjuvant trials of prostate cancer therapies for primary radiation therapy-based trials. Research is ongoing and needed to quantify other clinical benefits from preventing a PSA relapse in this setting.

quintessential end point of overall survival (OS) is used as the primary end point, (neo)adjuvant prostate cancer clinical trials usually take longer than a decade to be reported.

Recently, the Intermediate Clinical Endpoints in Cancer of the Prostate (ICECaP) working group has shown that metastasis-free survival (MFS: metastasis on conventional imaging or death from any cause) is a strong surrogate for OS in localized prostate cancer trials. ${ }^{21}$ For new treatments with an anticipated treatment effect size that will decrease the rate of metastases by $>33 \%$ (ie, hazard ratio [HR] $<0.67)$, MFS as the primary end point can shorten the time to show an OS benefit. ${ }^{21}$ Disease-free survival (DFS), which is MFS plus local-regional recurrence events, is not as strong a surrogate for OS.

Use of an earlier intermediate clinical end point (ICE) including prostate-specific antigen (PSA) recurrence could result in even more expeditious adjuvant trial conduct than DFS or MFS. However, for an ICE to serve as a good surrogate for OS, there must be no confounding from salvage therapy for relapsed disease that can either cure the disease and/or prolong postrelapse survival to the point that competing risks of death affect the analysis. ${ }^{22-27}$ Salvage prostate bed radiation after a prostatectomy and the prolonged survival of patients treated with androgen deprivation therapy (ADT) for biochemical recurrence (BCR) are potential confounders for prostate cancer outcomes. Prior studies have shown PSA nadir, end of treatment PSA, time to BCR, and post-BCR PSA doubling time (PSA-DT), are prognostic for OS and have some surrogacy at the patient level. ${ }^{28-31}$ However, to our knowledge, no prior studies using individual patient data (IPD) from multiple studies have been conducted to assess trial-level surrogacy, which evaluates whether a treatment effect on the ICE (eg, time to BCR) is correlated with the treatment effect on the ultimate end point (ie, OS) across many trials.
We hypothesized that event-free survival (EFS), which includes all components of DFS plus PSA-only relapse, may be a surrogate for OS at both the patient and the trial level. We also assessed the surrogacy of EFS for MFS, because MFS is a strong surrogate for OS. Given the observation that most patients with intermediate- or high-risk localized prostate cancer are cured, and even if they relapse with a rising PSA, often die as a result of a nonprostate cancer death, we also investigated the surrogacy of ICEs for disease-specific survival (DSS).

\section{METHODS}

\section{Trial Selection Criteria}

Eligible trials were identified from the established ICECaP data repository, composed of 22,825 IPD from 28 randomized trials conducted in Australia, Canada, Europe, New Zealand, and the United States for localized prostate cancer. ${ }^{1}$ For this analysis, eligible trials included radiation therapy as primary therapy that had evaluable PSA and disease follow-up data suitable for EFS analysis (see end point definitions). Prostatectomy-based trials were not included because of a lack of evaluable PSA data.

\section{Definition of End Points}

EFS was measured from the date of random assignment to the date of first evidence of disease recurrence, including biochemical failure, local or regional recurrence, distant metastasis, or death from any cause, or was censored at the date of last PSA assessment. Death without prior disease recurrence was also censored at the last PSA assessment if the interval between the last PSA assessment and the date of death was $>15$ months. MFS was measured from the date of random assignment to the date of the first evidence of recorded distant metastases or death from any cause, or was censored at the date of last follow-up. OS was measured from the date of random assignment to death from any cause and censored at the date of last follow-up in 


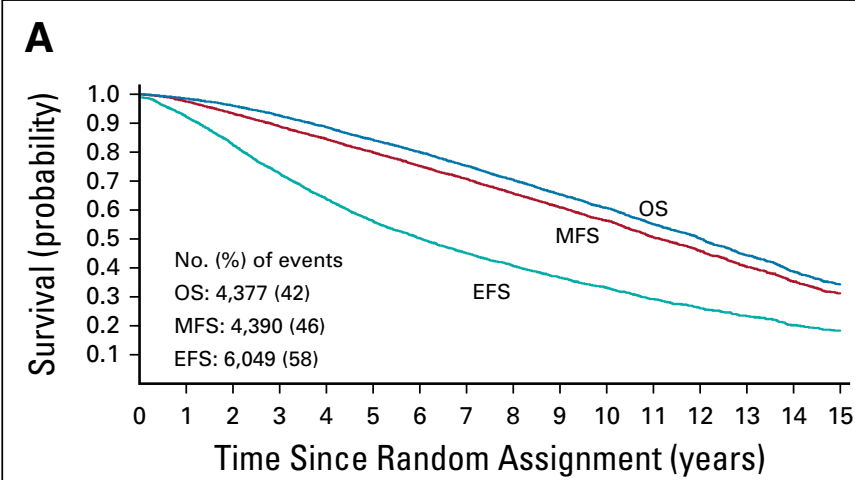

\section{B}
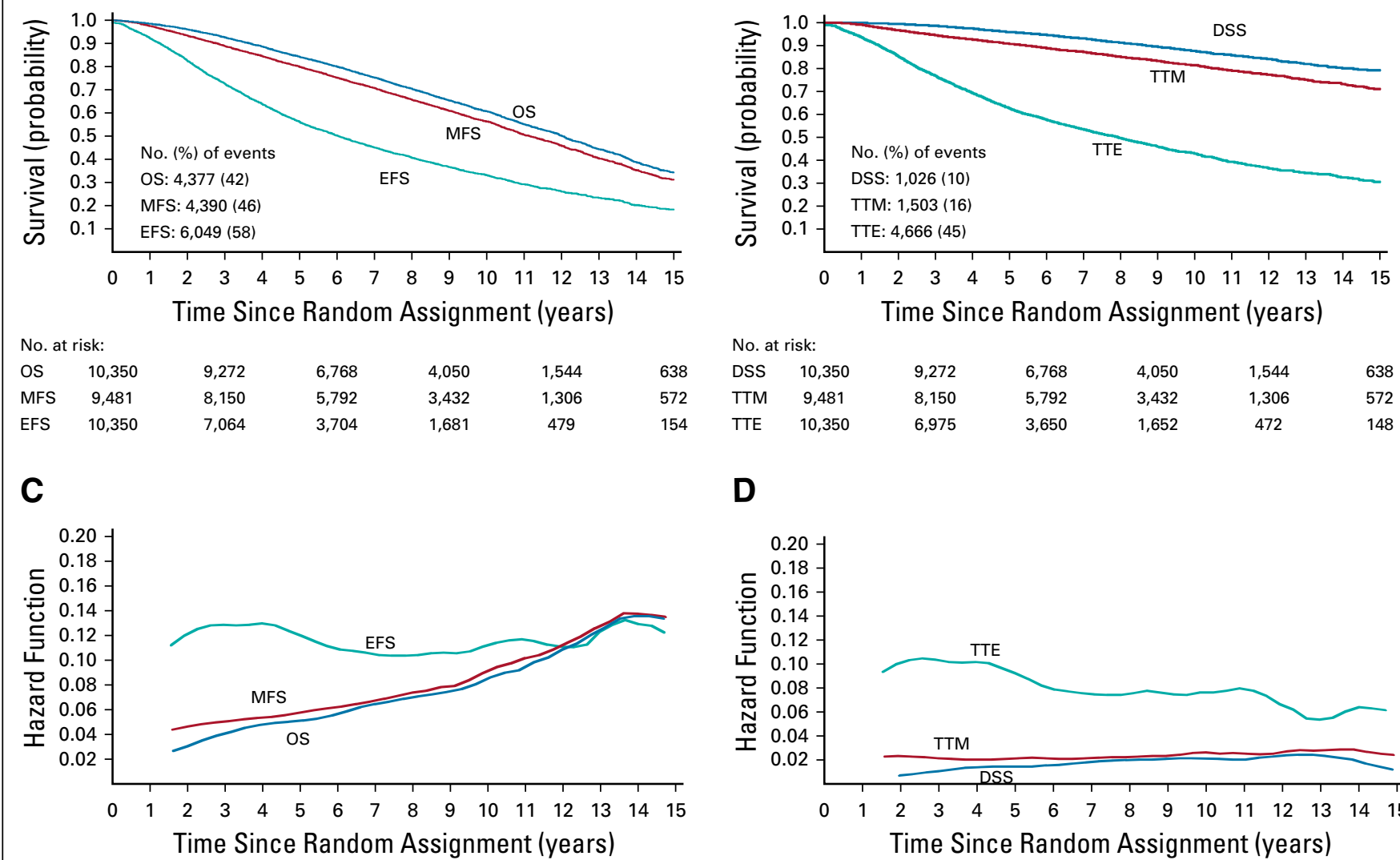

No. at risk:

$\begin{array}{lcccccc}\text { DSS } & 10,350 & 9,272 & 6,768 & 4,050 & 1,544 & 638 \\ \text { TTM } & 9,481 & 8,150 & 5,792 & 3,432 & 1,306 & 572 \\ \text { TTE } & 10,350 & 6,975 & 3,650 & 1,652 & 472 & 148\end{array}$

D

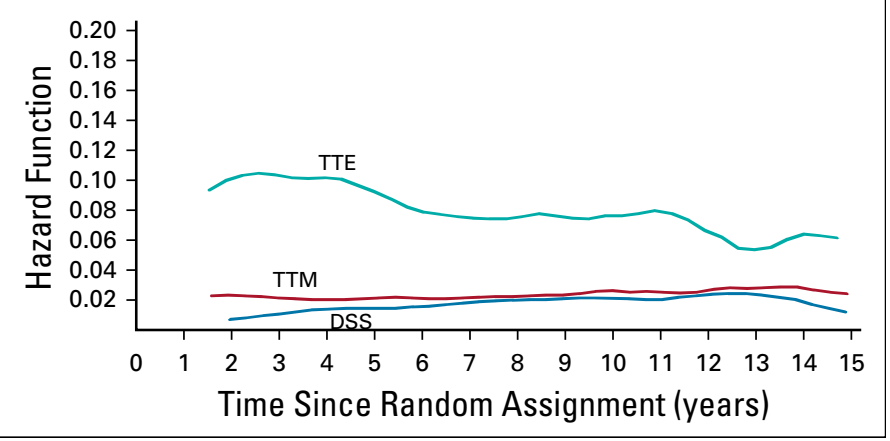

FIG 1. (A) Kaplan-Meier estimates of overall survival (OS), metastasis-free survival (MFS), and event-free survival (EFS) where non-prostate cancer deaths were counted as events. (B) Kaplan-Meier estimates of disease-specific survival (DSS), time to metastasis (TTM), and time to event (TTE) where non-prostate cancer deaths were censored. (C) Estimated hazard functions over time for OS, MFS, and EFS. (D) Estimated hazard functions over time for DSS, TTM, and TTE. Median follow-up was 10 years.

surviving patients. Time to event (TTE), time to metastasis (TTM), and DSS were defined analogously to EFS, MFS, and OS, respectively, but patients with nonprostate deaths had end points censored or considered as competing risk in sensitivity analyses.

Biochemical failure was defined according to the 2006 RTOG-ASTRO Phoenix definition. A PSA rise by $\geq 2 \mathrm{ng} / \mathrm{mL}$ above the nadir was considered biochemical failure; patients not fully meeting the PSA criteria for failure who underwent subsequent therapy were also declared to be failures at the time of salvage initiation. Of the 15 included trials, 14 trials were viable for harmonization by the Phoenix definition and 1 trial used the ASTRO definition (Data Supplement, online only). Local or regional recurrence was based on trial-defined events; distant metastasis was confirmed by imaging or histologic evidence as described in our prior MFS analysis. ${ }^{21}$

\section{Statistical Analyses}

Surrogacy criteria. We evaluated the surrogacy of EFS with OS using a 2-stage meta-analytic validation model as described previously. ${ }^{32}$ Two conditions must hold to claim
EFS is a surrogate for OS. Condition 1 requires EFS and OS to be correlated. Condition 2 requires that the treatment effects on both end points be correlated. The validity of the surrogate is reflected by the strength of both correlations. To be consistent with our previous work and other surrogacy assessments in oncology, we defined a priori a clinically relevant surrogacy as $R^{2} \geq 0.7 .1,21$

Condition 1 was tested at both the patient and the trial level. At the patient level, associations of OS with EFS were evaluated via a bivariate copula model over the entire follow-up of IPD (Data Supplement). Kendall's tau (range, 0-1) quantified the correlation between end points. At the trial level, we first obtained Kaplan-Meier estimates of 5-year EFS and 8-year OS rates for each treatment arm within each trial. We then performed weighted linear regression (WLR) of trial- and arm-specific 8-year OS rates on 5-year EFS rates. Similar analyses were performed at an earlier milestone time: OS rates at 5 years on EFS rates at 3 years. These time points were chosen because they are reported frequently in the literature and reflect the earlier time of biochemical failures. Regressions were weighted by 


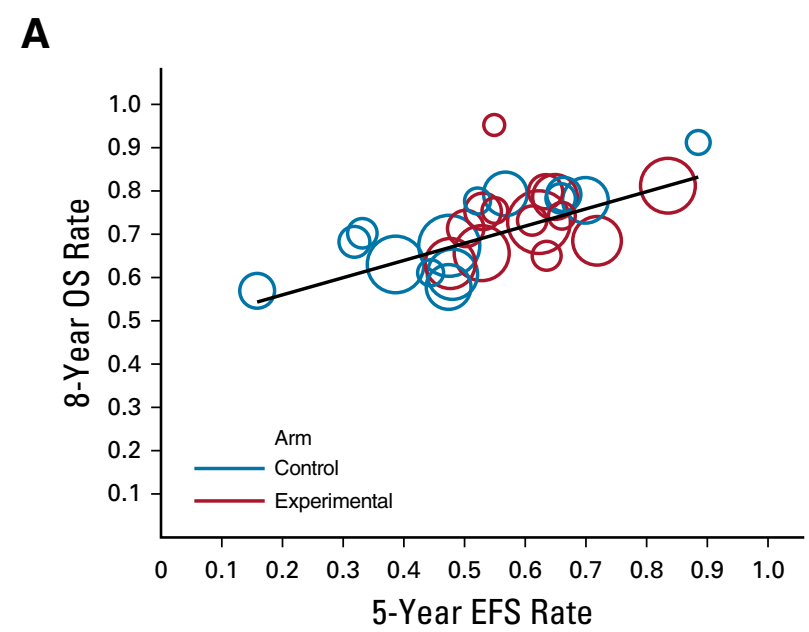

B

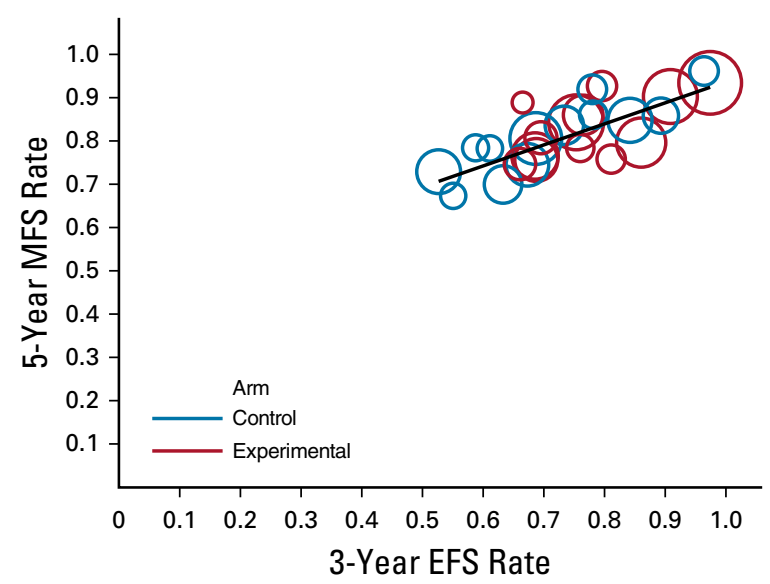

C

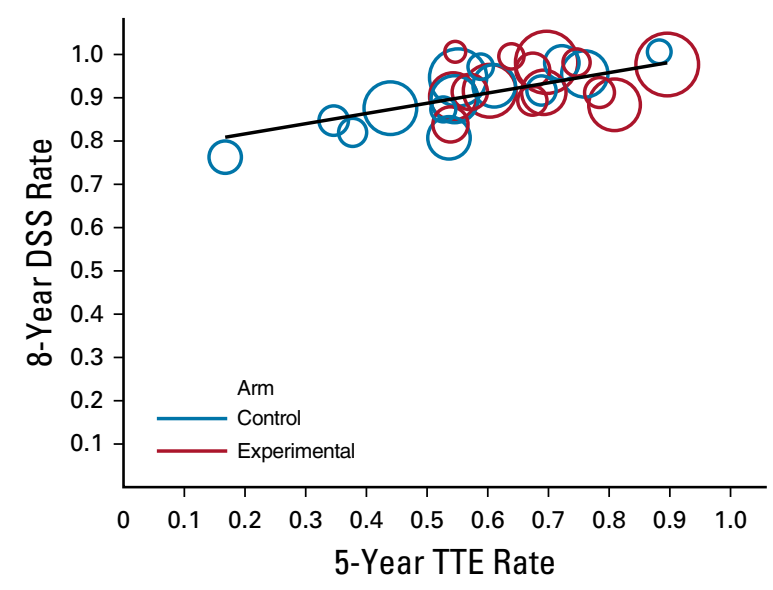

D

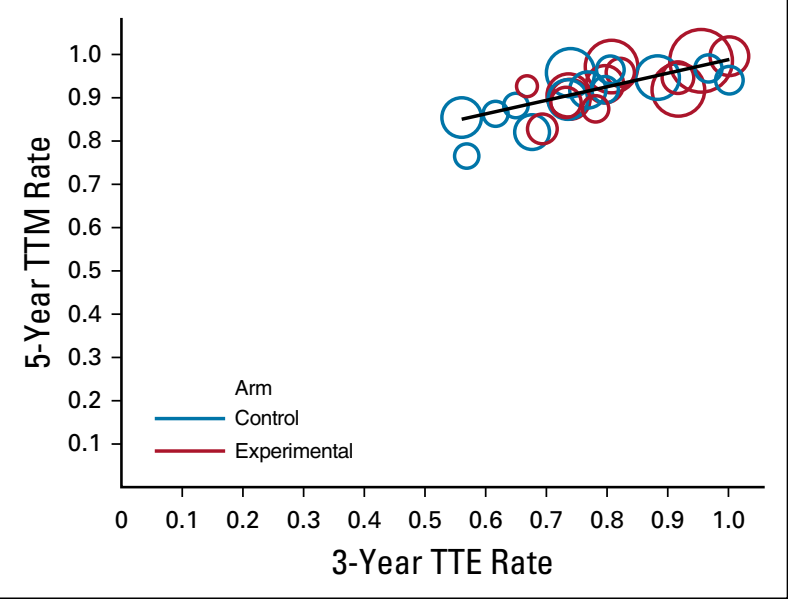

FIG 2. Bubble plot and regression of (A) overall survival (OS) at 8 years on event-free survival (EFS) at 5 years; (B) metastasis-free survival (MFS) at 5 years on EFS at 3 years; (C) disease-specific survival (DSS) at 8 years on time to event (TTE) at 5 years; and (D) time to metastasis (TTM) at 5 years on TTE at 3 years. All rates were Kaplan-Meier estimates by trial and treatment arm. Circle size and regression were weighted by inverse variance of the 5- or 3-year estimate for EFS and TTE.

the inverse of variances of the 5- or 3-year estimates of EFS. $R^{2}$ was used to quantify the proportion of variance that was explained by the regressions.

Condition 2 was tested at the trial level. Proportional hazards models estimated the study-specific treatment effects (ie, the natural $\log [\mathrm{HR}]$ of the experimental $v$ the control arm) on the EFS and OS. We then fit a WLR of $\log (H R)-O S$ on $\log (\mathrm{HR})$-EFS across trials. Regressions were weighted by inverse variances of the $\log (\mathrm{HR})$-EFS. The same approach was applied to the surrogacy analysis of EFS for MFS, and the surrogacy of TTE for TTM and DSS, where end points of patients with nonprostate cancer deaths were censored.

Subgroup and sensitivity analysis. We conducted preplanned subgroup analyses by age ( $<70$ years, $\geq 70$ years), by duration of ADT (None, 3-8 months, $\geq 2$ years), by patient risk groups (defined by the National Comprehensive Cancer Network [NCCN] or D'Amico's criteria), and by biochemical failure criteria (Phoenix definition only). Because a large proportion of OS events were non-prostate cancer deaths, we performed sensitivity analyses to estimate the trial-level correlation between cumulative incidence estimates of TTE and DSS, and between the subdistribution treatment effect HR estimates for TTE and DSS from competing risk models, for which non-prostate cancer deaths were considered to be the competing risk for each end point. Model accuracy was assessed by the leave-one-out cross validation approach (Data Supplement).

Surrogate threshold effect. The surrogate threshold effect (STE) is defined as the minimum treatment effect on the surrogate (HR-EFS) necessary to predict a significant OS benefit, corresponding to the upper 95\% prediction limit for OS HR lower than 1 (Data Supplement). All analyses were performed using SAS 9.4 (SAS Institute, Cary, NC) and R packages. ${ }^{33}$ 
TABLE 1. Surrogacy Condition 1: Correlation Between Clinical End Points (correlation using patient-level data) Correlation Using Patient-Level Data

No. of Trials

No. of Patients

Kendall's Tau (95\% Cl)

\begin{tabular}{llrr}
\hline When non-CaP deaths were counted as events & & & \\
\hline Correlation of OS and EFS & 16 & 10,350 & 0.43 (0.42 to 0.44) \\
\hline Correlation of MFS and EFS & $13^{\mathrm{a}}$ & 9,481 & 0.51 (0.49 to 0.52) \\
\hline When non-CaP deaths were censored & & & 0.52 (0.50 to 0.54$)$ \\
\hline Correlation of DSS and TTE & $14^{\mathrm{b}}$ & 9,934 & $0.53(0.51$ to 0.55$)$
\end{tabular}

Abbreviations: CaP, cancer of the prostate; DSS, disease-specific survival; EFS, event-free survival; MFS, metastasis-free survival; OS, overall survival; TTE, time to event; TTM, time to metastasis.

aExcluding 3 studies that did not collect metastasis data.

bexcluding 2 studies with $<3$ prostate cancer deaths.

\section{RESULTS}

\section{Trial and Patient Characteristics}

For analysis, 10,350 patients from 15 primary radiation therapy-based trials were included (Data Supplement). One trial was split into 2 experimental arms, which resulted in 16 study units for EFS and OS analysis and 13 study units for MFS analysis (Data Supplement). Most trials compared the duration of ADT (10 trials) or different radiation doses or fields (4 trials). Patients enrolled in the trials from 1987 to 2011, and the median follow-up was 10 years (range, 0.122 years). More than $80 \%$ of patients were $<75$ years of age, and nearly $60 \%$ had high-risk disease according to the NCCN or D'Amico criteria (Data Supplement).

For the EFS end point, 6,049 events were reported, 4,666 (77\%) from disease recurrence (biochemical failure only $56 \%$; the following were with or without reported biochemical failure: local or regional recurrence, $12 \%$; distant metastasis, 6\%; and unknown recurrence site, 3\%) and $23 \%$ from nonprostate cancer death (Data Supplement). The median duration of EFS from random assignment was
6.0 years ( $95 \% \mathrm{Cl}, 5.9$ to 6.2 years). There were 4,390 events for the MFS end point: $29 \%$ metastasis, $5 \%$ prostate cancer death without recoded metastasis, and $66 \%$ non-prostate cancer death. Observed 5-year rates from the Kaplan-Meier curves were $56 \%(95 \% \mathrm{Cl}, 55 \%$ to $57 \%)$ for EFS, $80 \%(95 \% \mathrm{Cl}, 79 \%$ to $81 \%)$ for MFS, and $84 \%(95 \% \mathrm{Cl}$, $84 \%$ to $85 \%$ ) for OS (Fig 1). Cumulative event rates for disease recurrence, metastasis, and prostate cancer death compared with non-prostate cancer death were delineated for the overall cohort and by the NCCN risk group and age group (Data Supplement). For patients who experienced an EFS disease recurrence event ( $n=4,666$ of the 6,049 events), median postrecurrence OS was 7.4 years $(95 \% \mathrm{Cl}, 7.1$ to 7.7 years). For patients who were reported to have a metastasis ( $n=1,287$ of 4,390 MFS events), median postmetastasis survival was 1.9 years $(95 \% \mathrm{Cl}, 1.8$ to 2.1 years).

\section{Surrogacy Condition 1: Correlation Between ICE and OS}

At the patient level, the Kendall's tau correlation over the entire follow-up was 0.43 ( $95 \% \mathrm{Cl}, 0.42$ to 0.44 ) for EFS with OS and $0.51(95 \% \mathrm{Cl}, 0.49$ to 0.52$)$ for EFS with MFS. When nonprostate cancer deaths were censored, the correlations of TTE

TABLE 2. Surrogacy Condition 1: Correlation Between Clinical End Points (regression using trial level estimates)

\begin{tabular}{|c|c|c|c|}
\hline Regression Using Trial-Level Estimates & No. of Trials & No. of Arms & $R^{2}(95 \% \mathrm{Cl})$ \\
\hline \multicolumn{4}{|c|}{ When non-CaP deaths were counted as events } \\
\hline 8-year OS rate on 5-year EFS rate & $14^{\mathrm{b}}$ & 28 & $0.55(0.26$ to 0.70$)$ \\
\hline 8-year MFS rate on 5-year EFS rate & $11^{\mathrm{a}, \mathrm{b}}$ & 22 & $0.63(0.31$ to 0.76$)$ \\
\hline 5-year OS rate on 3-year EFS rate & 16 & 32 & 0.61 (0.35 to 0.73$)$ \\
\hline 5-year MFS rate on 3-year EFS rate & $13^{\mathrm{a}}$ & 26 & $0.73(0.48$ to 0.82$)$ \\
\hline \multicolumn{4}{|l|}{ When non-CaP deaths were censored } \\
\hline 8-year DSS on 5-year TTE rate & $14^{\mathrm{b}}$ & 28 & $0.45(0.15$ to 0.63$)$ \\
\hline 8-year TTM on 5-year TTE rate & $11^{\mathrm{a}, \mathrm{b}}$ & 22 & $0.49(0.15$ to 0.67$)$ \\
\hline 5-year DSS on 3-year TTE rate & 16 & 32 & $0.44(0.17$ to 0.62$)$ \\
\hline 5-year TTM on 3-year TTE rate & $13^{\mathrm{a}}$ & 26 & $0.57(0.27$ to 0.72$)$ \\
\hline
\end{tabular}

Abbreviations: CaP, cancer of the prostate; DSS, disease-specific survival; EFS, event-free survival; MFS, metastasis-free survival; OS, overall survival; TTE, time to event; TTM, time to metastasis.

aExcluding 3 studies that did not collect metastasis data.

${ }^{\text {b}}$ Excluding 2 studies with median follow-up of $<6$ years. 
with DSS and with TTM were $0.52(95 \% \mathrm{Cl}, 0.50$ to 0.54$)$ and 0.53 (95\% Cl, 0.51 to 0.55), respectively.

The end point correlation was also tested using trial-level estimates (Fig 2). The $R^{2}$ was $0.55(95 \% \mathrm{Cl}, 0.26$ to 0.70 ) from WLR of 8-year OS on 5-year EFS rates and was 0.63 ( $95 \% \mathrm{Cl}, 0.31$ to 0.76 ) of 8-year MFS on 5-year EFS rates across trials and treatment arms. With earlier milestone time points of 5-year OS and MFS rates versus 3-year EFS rates, $R^{2}$ was $0.61(95 \% \mathrm{Cl}, 0.35$ to 0.73$)$ and 0.73 (95\% Cl, 0.48 to 0.82), respectively. Tables 1 and 2 also summarize $R^{2}$ for these end points when non-prostate cancer deaths were censored.

\section{Surrogacy Condition 2: Correlation Between Treatment Effect on ICE and OS}

At the trial level, trial-specific treatment effects, measured by log-HR for each end point, are shown in forest plots in the Data Supplement. The $R^{2}$ from the WLR of $\log (\mathrm{HR})$-OS on $\log (\mathrm{HR})$-EFS was $0.35(95 \% \mathrm{Cl}, 0.01$ to 0.60$)$ and was $0.55(95 \% \mathrm{Cl}, 0.09$ to 0.74$)$ for $\log (\mathrm{HR})-\mathrm{MFS}$ on $\log (\mathrm{HR})$ EFS (Table 3). When non-prostate cancer deaths were censored, the $R^{2}$ of $\log (\mathrm{HR})$-DSS on $\log (\mathrm{HR})$-TTE was 0.39 $(95 \% \mathrm{Cl}, 0.01$ to 0.64$)$ and was $0.68(95 \% \mathrm{Cl}, 0.23$ to 0.81$)$ for $\log (\mathrm{HR})$-TTM on $\log (\mathrm{HR})$-TTE (Fig 3).

\section{Subgroup and Sensitivity Analysis}

At the patient level, results were consistent when the analysis was restricted to the 14 trials using the Phoenix definition for biochemical failure, or to the populations with high-risk features or above and below the median age of 70 years (Data Supplement). The Kendall's tau correlation between OS and EFS was slightly stronger in those who received $\geq 2$ years of ADT compared with those who received short-term or no (neo)adjuvant ADT $(0.53,0.43$, and 0.39 , respectively). In patients with high-risk features and when non-prostate cancer deaths were censored, the estimated $R^{2}$ from the WLR of $\log (\mathrm{HR})$-DSS on $\log (\mathrm{HR})-\mathrm{TTE}$ across trials was 0.64 ( $95 \% \mathrm{Cl}, 0.21$ to 0.79 ). Results were also consistent in a WLR analysis of trial-level correlations when nonprostate cancer deaths were treated as competing risk
(Data Supplement) and in leave-one-out cross-validation (Data Supplement).

\section{STE}

The STE was an HR(EFS) of 0.33 on OS (Fig $3 A$ ) and an $\mathrm{HR}(\mathrm{EFS}$ ) of 0.44 on MFS (Fig 3B), which indicates that large risk reductions of at least $67 \%$ and $56 \%$ on EFS would predict a significant treatment effect on OS and MFS, respectively. The STE was an HR(TTE) of 0.29 on DSS (Fig 3C) and an HR(TTE) of 0.48 on TTM (Fig 3D) when non-prostate cancer deaths were censored.

\section{DISCUSSION}

This work clearly shows that EFS is a weak surrogate for both OS and DSS for men with intermediate- and high-risk localized prostate cancer treated with curative-intent radiation with a $10 \%$ chance of dying as a result of prostate cancer and approximately a 60\% OS at 10 years. Notably, this analysis included only patients treated with primary radiation, and therefore, the findings should not be extrapolated to trials of patients treated with prostatectomy or salvage radiation after prostatectomy ${ }^{26}$. As the estimated hazard curves over time (Fig 1) depict, there are many early EFS events (mostly PSA relapses) with few MFS and OS events. This indicates that despite $80 \%$ of men being younger than 75 years old and fit for a clinical trial, they are still more likely to die a non-prostate cancer death even if they have a PSA relapse. This provides additional evidence that many PSA relapses are indolent and/or are controlled by testosterone suppression. ${ }^{34,35}$

Prior studies have shown PSA relapse heralding a prostate cancer recurrence is prognostic for OS and DSS, mainly on the basis of single-trial, patient-level data and using landmark analysis at selected time points. ${ }^{29-31}$ In particular, the subpopulation of younger men with a rapidly rising PSA at relapse are more likely to die as a result of prostate cancer. However, the new data presented in this manuscript showing the low correlation of EFS with OS at both the patient and the trial level indicate that EFS is not a viable surrogate for OS to replace it as the primary end point in phase III localized prostate cancer trials. This was

TABLE 3. Surrogacy Condition 2: Treatment Effects on End Points are Correlated

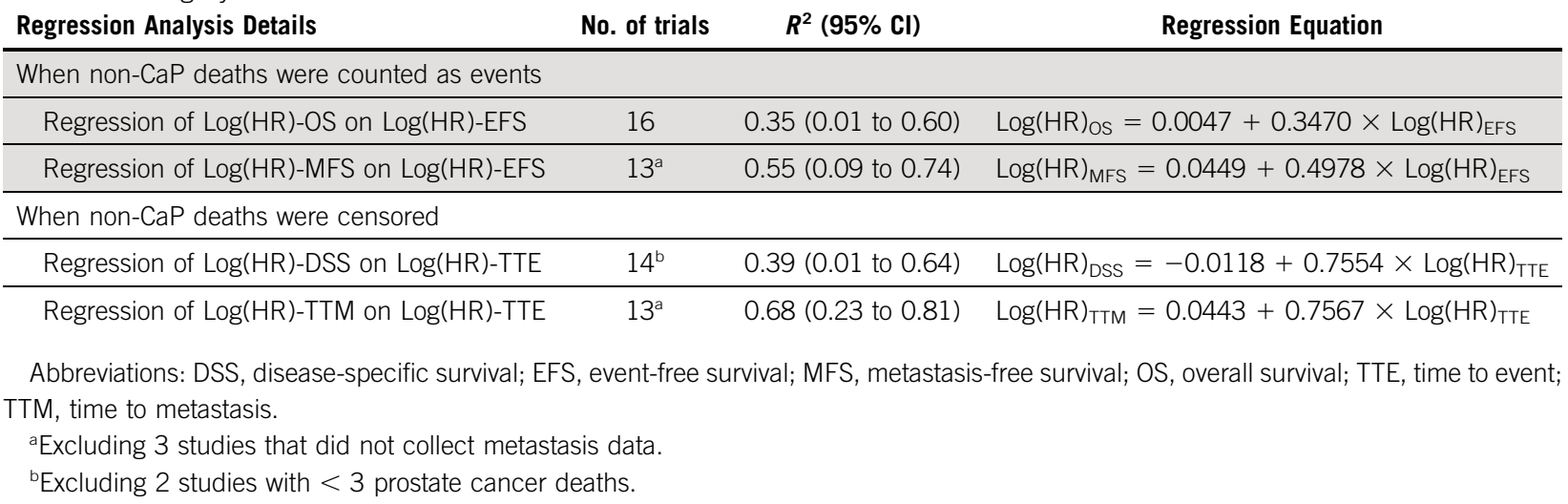



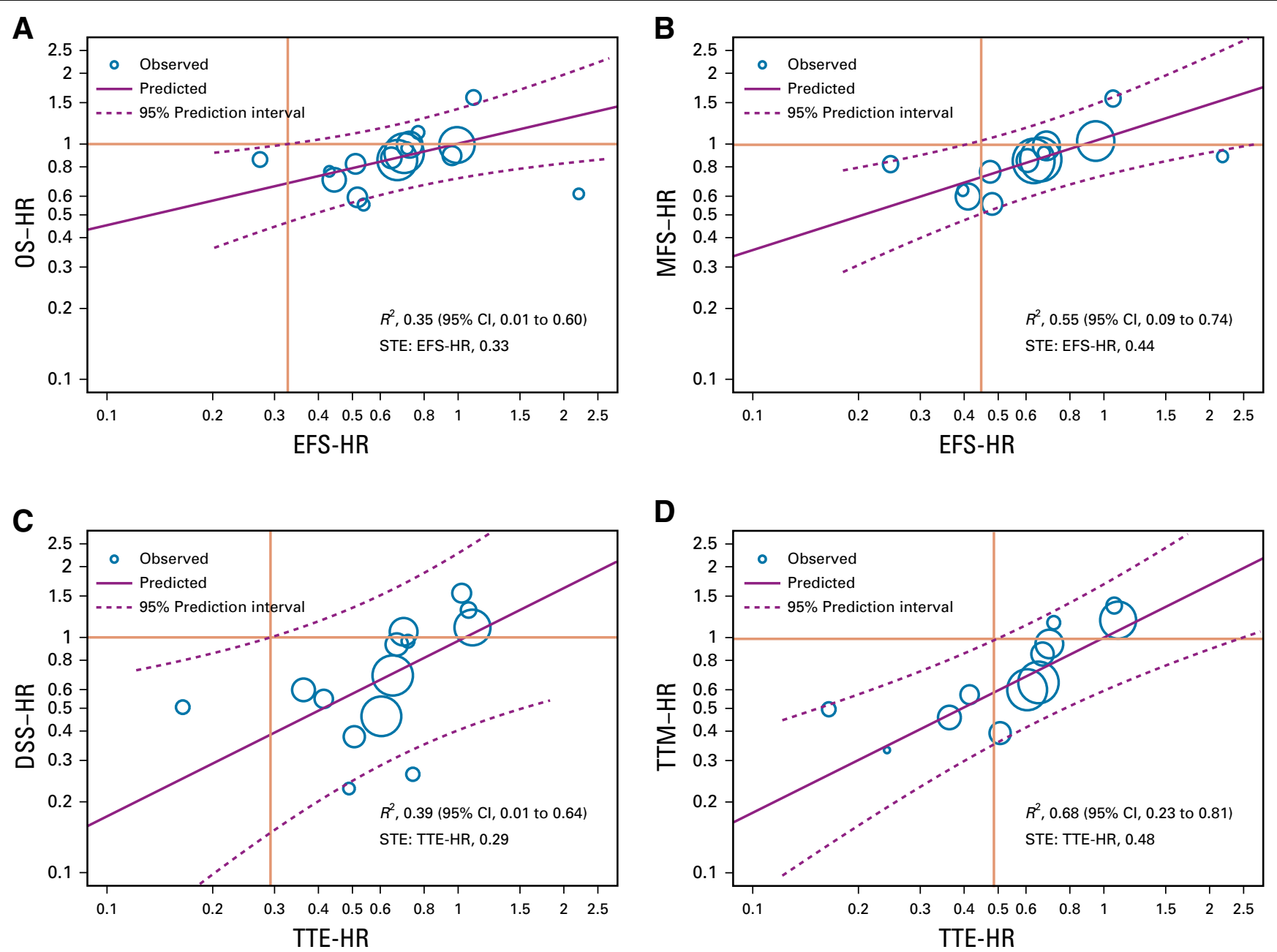

D

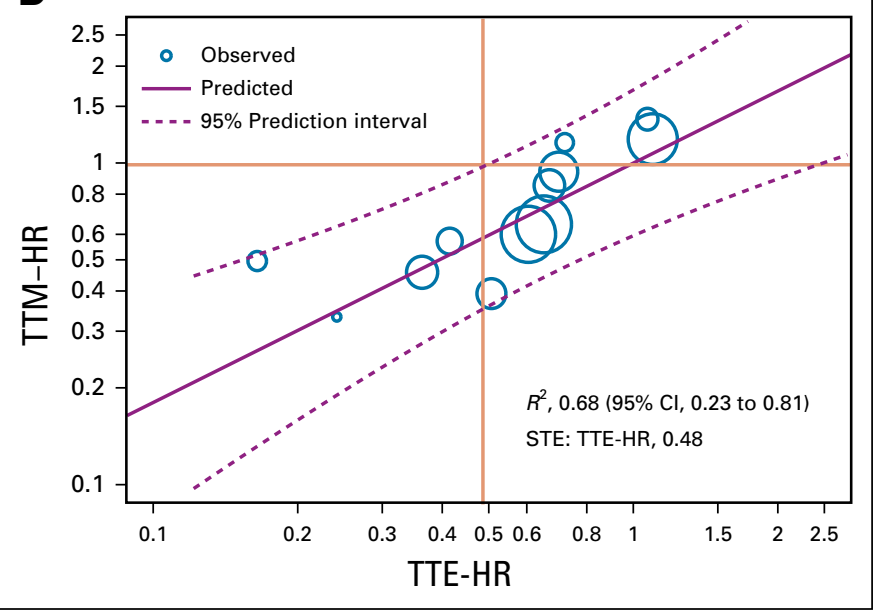

FIG 3. Bubble plot and regression of (A) hazard ratio for overall survival (OS-HR) on hazard ratio for event-free survival (EFS-HR); (B) hazard ratio for metastasis-free survival (MFS-HR) on EFS-HR; (C) hazard ratio for disease-specific survival (DSS-HR) on hazard ratio for time to event (TTE-HR); (D) hazard ratio for time to metastasis (TTM-HR) on TTE-HR. Cox proportional hazards regression estimated HR for each study, and values were natural logarithm transformed. Circle size and regression were weighted by inverse variance of $\log (\mathrm{HR})$ estimates for EFS or TTE. The orange lines display surrogate threshold effect (STE), which is the intersection of the upper 95\% prediction limit with the horizontal line representing an HR of 1 for the true end points.

underscored by showing that removal of non-prostate cancer deaths (approximately $20 \%$ of EFS events) as part of the TTE analysis did not substantially improve the correlation. By contrast, MFS was a strong surrogate for OS in the same population treated with radiation (with all surrogacy association parameters $>0.8) .{ }^{21}$ EFS may play a role in enriching the analysis or the interim monitoring analysis during phase III clinical trials. ${ }^{36}$ This finding does not undermine the observation that identification of PSA relapses has other benefits such as identifying men for postprostatectomy salvage radiation and use of PSA-DT for counseling about prognosis. The long-term results of ongoing (neo)adjuvant trials with potent androgen receptor inhibitors will determine whether EFS can serve as a surrogate for OS in this setting. This possibility is further confounded by the fact this class of therapy may directly affect the biomarker (PSA).
It is also recognized that there are other benefits to patients and society from not having a PSA relapse. From the patient perspective, there is the potential impairment in quality of life associated with a PSA relapse, both from the anxiety of the recurrence itself and from the adverse effects of salvage radiation therapy (if treated with a prostatectomy) and protracted testosterone suppression, noting the median postrecurrence survival in this cohort was 7.4 years. In addition, there are the patient and health care impacts from increasing comorbidities associated with prolonged ADT such as glucose intolerance, cardiovascular events, and bone loss. If a patient does progress to having metastatic disease, there are additional impairments from cancer-related events of bone pain and fatigue and the costs and toxicities of more potent hormonal therapy and cytotoxic agents. ${ }^{37}$ Recent modeling analyses from the STAMPEDE docetaxel results show that preventing progression events with docetaxel was 
also a more efficient use of health care resources. ${ }^{38}$ The ICECaP Working Group has established a health economics team who are using the ICECaP IPD to estimate the health economic impacts on patients and society of preventing metastatic disease and earlier PSA relapse. If these benefits of preventing earlier relapses can be defined at both the patient and the societal level, and they offset the associated costs from the use of the new therapies in the (neo)adjuvant setting, then PSA-based end points could be used to accelerate the conduct of adjuvant prostate cancer trials.

The inherent limitations of this work are as follows. First, the data were generated from an era before the use of the more active therapies shown to prolong OS in the metastatic hormone-sensitive prostate cancer and CRPC settings. ${ }^{6-9,17,18}$ However, a recent analysis indicates that the cumulative use and efficacy of these new therapies have a limited impact on CRPC longevity. ${ }^{39}$ It is hoped that some of these new therapies will have more impact in the adjuvant setting. Second, because of incomplete PSA or salvage therapies, only a subset of all the trials in the ICECaP database was included in the EFS analysis, and these were

\section{AFFILIATIONS}

${ }^{1}$ Division of Biostatistics, Dana-Farber Cancer Institute, Boston, MA

${ }^{2}$ International Drug Development Institute, Louvain la Neuve, Belgium ${ }^{3}$ Department of Biostatistics and Bioinformatics, Duke University,

Durham, NC

${ }^{4}$ Department of Medicine, Memorial Sloan-Kettering Cancer Center, New York, NY

${ }^{5}$ Departments of Medicine \& Urology, Tulane University, New Orleans, LA

${ }^{6}$ Prostate Cancer Foundation, Santa Monica, CA

${ }^{7}$ Department of Biostatistics, The University of Texas MD Anderson

Cancer Center, Houston, TX

${ }^{8}$ Urological Oncology, The Christie NHS Foundation Trust, Manchester, United Kingdom

${ }^{9}$ European Organisation for Research and Treatment of Cancer Headquarters, Brussels, Belgium

${ }^{10}$ Department of Radiation Oncology, Brigham and Women's Hospital and Dana-Farber Cancer Institute, Boston, MA

${ }^{11}$ Centre for Health Economics Research and Evaluation, University of Technology Sydney, Sydney, NSW, Australia

${ }^{12}$ Department of Public Health Science, University of Chicago, Chicago, IL

${ }^{13}$ Sidney Kimmel Comprehensive Cancer Center, Johns Hopkins University, Baltimore, MD

${ }^{14}$ University Hospitals Birmingham, Birmingham, United Kingdom

${ }^{15}$ Department of Cancer Medicine, Institut Gustave Roussy, Villejuif, France

${ }^{16}$ Division of Cancer Sciences, University of Manchester and The Christie, Manchester, United Kingdom

${ }^{17}$ Urology Oncology, University Jean Monnet, St Etienne, France

${ }^{18}$ Canadian Cancer Trials Group, Cancer Research Institute, Queen's University, Kingston, Ontario, Canada

${ }^{19}$ Radiation Oncology, Cedars Sinai Medical Center, Los Angeles, CA

${ }^{20}$ University of Michigan, Ann Arbor, MI

${ }^{21}$ Medical Research Council Clinical Trials Unit, Institute of Clinical Trials and Methodology, University College London, London, United Kingdom

${ }^{22}$ Institut de Recherche Clinique, Université Catholique de Louvain, Brussels, Belgium only radiation-based trials. However, the analyzed IPD from 10,000 patients was representative of both the entire ICECaP database (Data Supplement) and the general population of patients with intermediate- or high-risk prostate cancer treated with radiation, ${ }^{1}$ which supports the relevance of the results. Another notable limitation is the lack of granular testosterone and PSA data to assess doubling time before subsequent therapies; this highlights the need for a harmonized and strategic data collection plan for future trials to robustly assess other PSA metrics. ${ }^{40}$

In conclusion, EFS is only a weak surrogate for OS in clinically localized prostate cancer in a patient population treated with radiation with approximately a $10 \%$ chance of dying of prostate cancer over 10 years despite potentially curative local therapy. As such, EFS cannot be used as a surrogate for OS and replace it as the primary end point to accelerate (neo)adjuvant prostate cancer phase III trials. Health economic analyses are underway to determine if other metrics of benefits to patients and society can be quantified to support the use of EFS as an end point to accelerate (neo)adjuvant prostate cancer trials.

${ }^{23}$ Radiation Oncology, Peter MacCallum Cancer Centre, Melbourne, Australia

${ }^{24}$ Lank Center for Genitourinary Oncology, Dana-Farber Cancer Institute, Boston, MA

\section{CORRESPONDING AUTHOR}

Christopher J. Sweeney, MBBS, Dana-Farber Cancer Institute, 450

Brookline Ave, Boston, MA 02215; e-mail: christopher_sweeney@ dfci.harvard.edu.

\section{SUPPORT}

Supported by a Prostate Cancer Foundation Challenge Award and by grants from Astellas/Pfizer, Janssen, Takeda, Sotio, Sanofi, Bayer, and Dendreon.

\section{AUTHORS' DISCLOSURES OF POTENTIAL CONFLICTS OF INTEREST AND DATA AVAILABILITY STATEMENT}

Disclosures provided by the authors and data availability statement (if applicable) are available with this article at DOI https://doi.org/10.1200/ JC0.19.03114.

\section{AUTHOR CONTRIBUTIONS}

Conception and design: Wanling Xie, Meredith M. Regan, Marc Buyse, Susan Halabi, Oliver Sartor, Howard Soule, Mario Eisenberger, Nicholas James, Silke Gillessen, Wendy Parulekar, Bertrand Tombal, Scott Williams, Christopher J. Sweeney

Financial support: Howard Soule, Christopher J. Sweeney

Administrative support: Christopher J. Sweeney

Provision of study material or patients: Noel Clarke, Anthony D'Amico, James Dignam, Nicholas James, Karim Fizazi, Nicolas Mottet, Wendy Parulekar, Matthew R. Sydes, Christopher J. Sweeney, ICECaP Working Group

Collection and assembly of data: Meredith M. Regan, Laurence Collette, James Dignam, Nicholas James, Karim Fizazi, Wendy Parulekar, Howard Sandler, Matthew R. Sydes, Bertrand Tombal, Christopher J. Sweeney Data analysis and interpretation: Wanling Xie, Meredith M. Regan, Marc Buyse, Susan Halabi, Philip W. Kantoff, Oliver Sartor, Donald Berry, Noel 
Clarke, Laurence Collette, Anthony D'Amico, Richard De Abreu Lourenco, James Dignam, Mario Eisenberger, Nicholas James, Karim Fizazi, Silke Gillessen, Yohann Loriot, Nicolas Mottet, Wendy Parulekar, Daniel E. Spratt, Matthew R. Sydes, Scott Williams, Christopher

J. Sweeney

Manuscript writing: All authors

Final approval of manuscript: All authors

Accountable for all aspects of the work: All authors

\section{ACKNOWLEDGMENT}

We thank Victoria Wong, Frontier Science \& Technology Research

Foundation, for computer programming. We also thank the Writing Committee and ICECaP Working Group for all of their contributions (see Appendix for details).

\section{REFERENCES}

1. Sweeney C, Nakabayashi M, Regan M, et al: The development of Intermediate Clinical Endpoints in Cancer of the Prostate (ICECaP). J Natl Cancer Inst 107: djv261, 2015

2. Torre LA, Bray F, Siegel RL, et al: Global cancer statistics, 2012. CA Cancer J Clin 65:87-108, 2015

3. Siegel RL, Miller KD, Jemal A: Cancer statistics, 2016. CA Cancer J Clin 66:7-30, 2016

4. Bolla M, Gonzalez D, Warde P, et al: Improved survival in patients with locally advanced prostate cancer treated with radiotherapy and goserelin. N Engl J Med 337:295-300, 1997

5. Denham JW, Steigler A, Lamb DS, et al: Short-term neoadjuvant androgen deprivation and radiotherapy for locally advanced prostate cancer: 10-year data from the TROG 96.01 randomised trial. Lancet Oncol 12:451-459, 2011

6. Kyriakopoulos $\mathrm{CE}$, Chen YH, Carducci MA, et al: Chemohormonal therapy in metastatic hormone-sensitive prostate cancer: Long-term survival analysis of the randomized phase III E3805 CHAARTED trial. J Clin Oncol 36:1080-1087, 2018

7. James ND, de Bono JS, Spears MR, et al: Abiraterone for prostate cancer not previously treated with hormone therapy. N Engl J Med 377:338-351, 2017

8. James ND, Sydes MR, Clarke NW, et al: Addition of docetaxel, zoledronic acid, or both to first-line long-term hormone therapy in prostate cancer (STAMPEDE): Survival results from an adaptive, multiarm, multistage, platform randomised controlled trial. Lancet 387:1163-1177, 2016

9. Fizazi K, Tran N, Fein L, et al: Abiraterone plus orednisone in metastatic, castration-sensitive prostate cancer. N Engl J Med 377:352-360, 2017

10. Fizazi K, Scher HI, Molina A, et al: Abiraterone acetate for treatment of metastatic castration-resistant prostate cancer: Final overall survival analysis of the COU-AA-301 randomised, double-blind, placebo-controlled phase 3 study. Lancet Oncol 13:983-992, 2012

11. Ryan CJ, Smith MR, Fizazi K, et al: Abiraterone acetate plus prednisone versus placebo plus prednisone in chemotherapy-naive men with metastatic castrationresistant prostate cancer (COU-AA-302): Final overall survival analysis of a randomised, double-blind, placebo-controlled phase 3 study. Lancet Oncol 16: 152-160, 2015

12. Beer TM, Armstrong AJ, Rathkopf DE, et al: Enzalutamide in metastatic prostate cancer before chemotherapy. N Engl J Med 371:424-433, 2014

13. de Bono JS, Oudard S, Ozguroglu M, et al: Prednisone plus cabazitaxel or mitoxantrone for metastatic castration-resistant prostate cancer progressing after docetaxel treatment: A randomised open-label trial. Lancet 376:1147-1154, 2010

14. Tannock IF, de Wit R, Berry WR, et al: Docetaxel plus prednisone or mitoxantrone plus prednisone for advanced prostate cancer. N Engl J Med 351:1502-1512, 2004

15. Parker C, Nilsson S, Heinrich D, et al: Alpha emitter radium-223 and survival in metastatic prostate cancer. N Engl J Med 369:213-223, 2013

16. Kantoff PW, Higano CS, Shore ND, et al: Sipuleucel-T immunotherapy for castration-resistant prostate cancer. N Engl J Med 363:411-422, 2010

17. Davis ID, Martin AJ, Stockler MR, et al: Enzalutamide with standard first-line therapy in metastatic prostate cancer. N Engl J Med 381:121-131, 2019

18. Chi KN, Agarwal N, Bjartell A, et al: Apalutamide for metastatic, castration-sensitive prostate cancer. N Engl J Med 381:13-24, 2019

19. Bolla M, Collette L, Blank L, et al: Long-term results with immediate androgen suppression and external irradiation in patients with locally advanced prostate cancer (an EORTC study): A phase III randomised trial. Lancet 360:103-106, 2002

20. D'Amico AV, Chen MH, Renshaw AA, et al: Androgen suppression and radiation vs radiation alone for prostate cancer: A randomized trial. JAMA 299:289-295, 2008

21. Xie W, Regan MM, Buyse M, et al: Metastasis-free survival is a strong surrogate of overall survival in localized prostate cancer. J Clin Oncol 35:3097-3104, 2017

22. Hamdy FC, Donovan JL, Lane JA, et al: 10-Year outcomes after monitoring, surgery, or radiotherapy for localized prostate cancer. N Engl J Med 375:1415-1424, 2016

23. Bill-Axelson A, Holmberg L, Garmo H, et al: Radical prostatectomy or watchful waiting in early prostate cancer. N Engl J Med 370:932-942, 2014

24. Crook JM, O'Callaghan CJ, Duncan G, et al: Intermittent androgen suppression for rising PSA level after radiotherapy. N Engl J Med 367:895-903, 2012 [Erratum: N Engl J Med 367:2262, 2012]

25. Ross AE, Yousefi K, Davicioni E, et al: Utility of risk models in decision making after radical prostatectomy: Lessons from a natural history cohort of intermediateand high-risk men. Eur Urol 69:496-504, 2016

26. Shipley WU, Seiferheld W, Lukka HR, et al: Radiation with or without antiandrogen therapy in recurrent prostate cancer. N Engl J Med 376:417-428, 2017

27. Broglio KR, Berry DA: Detecting an overall survival benefit that is derived from progression-free survival. J Natl Cancer Inst 101:1642-1649, 2009

28. Pound CR, Partin AW, Eisenberger MA, et al: Natural history of progression after PSA elevation following radical prostatectomy. JAMA 281:1591-1597, 1999

29. Dignam JJ, Hamstra DA, Lepor $\mathrm{H}$, et al: Time interval to biochemical failure as a surrogate end point in locally advanced prostate cancer: Analysis of randomized trial NRG/RTOG 9202. J Clin Oncol 37:213-221, 2019

30. Royce TJ, Chen MH, Wu J, et al: Surrogate end points for all-cause mortality in men with localized unfavorable-risk prostate cancer treated with radiation therapy vs radiation therapy plus androgen deprivation therapy: A secondary analysis of a randomized clinical trial. JAMA Oncol 3:652-658, 2017

31. D'Amico AV, Chen MH, de Castro M, et al: Surrogate endpoints for prostate cancer-specific mortality after radiotherapy and androgen suppression therapy in men with localised or locally advanced prostate cancer: An analysis of two randomised trials. Lancet Oncol 13:189-195, 2012

32. Ciani O, Davis S, Tappenden P, et al: Validation of surrogate endpoints in advanced solid tumors: Systematic review of statistical methods, results, and implications for policy makers. Int J Technol Assess Health Care 30:312-324, 2014

33. The R Project for Statistical Computing: www.r-project.org.

34. Freedland SJ, Humphreys EB, Mangold LA, et al: Death in patients with recurrent prostate cancer after radical prostatectomy: Prostate-specific antigen doubling time subgroups and their associated contributions to all-cause mortality. J Clin Oncol 25:1765-1771, 2007

35. Van den Broeck T, van den Bergh RCN, Arfi N, et al: Prognostic value of biochemical recurrence following treatment with curative intent for prostate cancer: A systematic review. Eur Urol 75:967-987, 2019 
36. Li Y, Taylor JM: Predicting treatment effects using biomarker data in a meta-analysis of clinical trials. Stat Med 29:1875-1889, 2010

37. Morgans AK, Stockler MR: Patient-reported outcomes in metastatic castration-sensitive prostate cancer in the adjuvant setting. Eur Urol Focus 5:144-146, 2019

38. Woods BS, Sideris E, Sydes MR, et al: Addition of docetaxel to first-line long-term hormone therapy in prostate cancer (STAMPEDE): Modelling to estimate longterm survival, quality-adjusted survival, and cost-effectiveness. Eur Urol Oncol 1:449-458, 2018

39. Francini E, Gray KP, Shaw GK, et al: Impact of new systemic therapies on overall survival of patients with metastatic castration-resistant prostate cancer in a hospital-based registry. Prostate Cancer Prostatic Dis 22:420-427, 2019

40. Kelloff GJ, Coffey DS, Chabner BA, et al: Prostate-specific antigen doubling time as a surrogate marker for evaluation of oncologic drugs to treat prostate cancer. Clin Cancer Res 10:3927-3933, 2004

\section{Looking for Trusted Patient Information?}

Direct your patients to Cancer.Net - ASCO's free patient education website.

- Up-to-date information

- Guides to more than 120 types of cancer

- Blog posts, videos, and podcasts

- Research summaries

- $\quad$ Survivorship care plans and more

Oncologist-Approved! Visit www.cancer.net today!
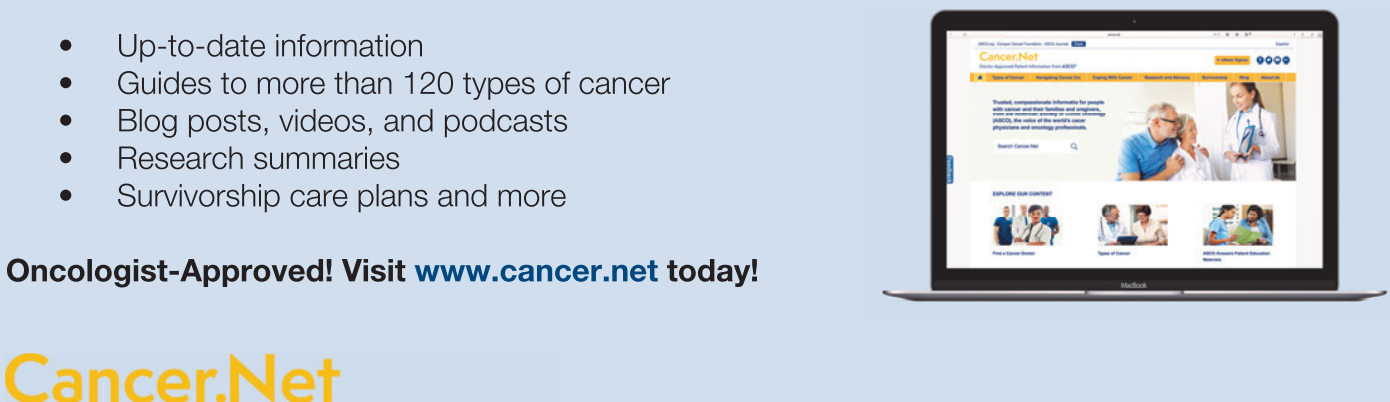

Doctor-Approved Patient Information from ASCO 
Event-Free Survival, a Prostate-Specific Antigen-Based Composite End Point, Is Not a Surrogate for Overall Survival in Men With Localized Prostate Cancer Treated With Radiation

The following represents disclosure information provided by authors of this manuscript. All relationships are considered compensated unless otherwise noted. Relationships are self-held unless noted. I = Immediate Family Member, Inst = My Institution. Relationships may not relate to the subject matter of this manuscript. For more information about ASCO's conflict of interest policy, please refer to www.asco.org/rwc or ascopubs.org/jco/authors/author-center.

Open Payments is a public database containing information reported by companies about payments made to US-licensed physicians (Open Payments).

\section{Meredith M. Regan}

Consulting or Advisory Role: Ipsen (Inst)

Research Funding: Veridex (Inst), OncoGenex (Inst), Pfizer (Inst), Ipsen (Inst), Novartis (Inst), Merck (Inst), Ferring (Inst), Celgene (Inst), AstraZeneca (Inst), Pierre Fabre (Inst), Ipsen (Inst), Bayer (Inst), Bristol-Myers Squibb (Inst), Roche (Inst), Astellas Pharma (Inst), Medivation (Inst), Janssen (Inst), Millenium Pharamceuticals (Inst), Sanofi (Inst), Sotio (Inst), Dendreon (Inst), Pfizer (Inst), TerSera (Inst)

Travel, Accommodations, Expenses: Bristol-Myers Squibb

Marc Buyse

Employment: IDDI

Stock and Other Ownership Interests: IDDI

Susan Halabi

Employment: ASCO Targeted Agent and Profiling Utilization Registry Consulting or Advisory Role: Eisai, Ferring Pharmaceuticals, Bayer

\section{Philip W. Kantof}

Leadership: Context Therapeutics

Stock and Other Ownership Interests: Placon, Druggablity Technologies, Context Therapeutics, Seer

Consulting or Advisory Role: Bavarian Nordic, Janssen, Merck, OncoCellMDX Genentech/Roche, Tarveda Therapeutics, Druggablity Technologies, Progenity, Context Therapeutics, GE Healthcare, Seer

Patents, Royalties, Other Intellectual Property: Method for Predicting the Risk of Prostate Cancer Morbidity and Mortality, Predicting and Treating Prostate Cancer, Methods for Predicting Likelihood of Responding to Treatment, Chromosome Copy Number Gain as a Biomarker of Urothelial Carcinoma Lethality, Drug Combinations to Treat Cancer, Somatic ERCC2 Mutations Correlate with Cisplatin sensitivity in muscle-invasive Urothelial Carcinoma (Patent), Up-to-Date Royalties, Wolters Kluwer Royalties, Methods and Kits for Determining Sensitivity to Cancer Treatment, Composition and Methods for Screening and Diagnosis of Prostate Cancer

Open Payments Link: https://openpaymentsdata.cms.gov/physician/55315/ summary

\section{Oliver Sartor}

Stock and Other Ownership Interests: Lilly, GlaxoSmithKline, AbbVie, Cardina Health, United Health Group, PSMA Therapeutics

Consulting or Advisory Role: Bayer, Johnson \& Johnson, Sanofi, AstraZeneca Dendreon, Endocyte, Constellation Pharmaceuticals, Advanced Accelerator Applications, Pfizer, Bristol-Myers Squibb, Bavarian Nordic, EMD Serono, Astellas Pharma, Progenics, Noxo, Blue Earth Diagnostics, Myovant, Myriad Genetics, Novartis, Clovis

Research Funding: Bayer (Inst), Johnson \& Johnson (Inst), Sanofi (Inst), Endocyte (Inst), Innocrin Pharma (Inst), Merck (Inst), InVitae (Inst), Constellation Pharmaceuticals (Inst), Advanced Accelerator Applications (Inst), AstraZeneca (Inst), Dendreon (Inst), Sotio

Expert Testimony: Sanofi

Travel, Accommodations, Expenses: Bayer, Johnson \& Johnson, Sanofi, AstraZeneca, Progenics

\section{Howard Soule}

Leadership: WindMIL

Consulting or Advisory Role: Compugen, WindMIL

Travel, Accommodations, Expenses: Compugen, Sanofi, WindMIL

\section{Donald Berry}

Employment: Berry Consultants

Leadership: Berry Consultants

Stock and Other Ownership Interests: Berry Consultants

Consulting or Advisory Role: Berry Consultants

Research Funding: Daiichi Sankyo

Travel, Accommodations, Expenses: Berry Consultants

Noel Clarke

Honoraria: Janssen-Cilag, Astellas Pharma, AstraZeneca

Speakers' Bureau: AstraZeneca, Astellas Pharma, Bayer, Ferring, Ipsen, Sanofi,

Amgen, Janssen

Research Funding: AstraZeneca (Inst)

Travel, Accommodations, Expenses: Ipsen, Janssen, Sanofi
Richard De Abreu Lourenco

Consulting or Advisory Role: Vifor Pharma (I)

James Dignam

Consulting or Advisory Role: Merck, Celgene, Northwest Biotherapeutics

\section{Mario Eisenberger}

Leadership: Veru

Stock and Other Ownership Interests: Veru

Honoraria: Merck Sharp \& Dohme, Bristol-Myers Squibb

Travel, Accommodations, Expenses: Veru

\section{Nicholas James}

Honoraria: Sanofi, Bayer, Oncogenex, Janssen, Astellas Pharma, Pierre Fabre Consulting or Advisory Role: Sanofi, Bayer, Astellas Pharma, Janssen, Clovis, EUSA Pharma, Pfizer

Speakers' Bureau: Pierre Fabre, Ferring, Sanofi, Astellas Pharma, Janssen Oncology

Research Funding: Janssen (Inst), Astellas Pharma (Inst), Pfizer (Inst), Sanofi (Inst), Novartis (Inst)

Travel, Accommodations, Expenses: Sanofi, Janssen

Karim Fizazi

Honoraria: Janssen, Sanofi, Astellas Pharma, Bayer

Consulting or Advisory Role: Janssen Oncology, Bayer, Astellas Pharma, Sanofi, Orion Pharma GmbH, Curevac (Inst), AstraZeneca (Inst), ESSA (Inst), Amgen (Inst)

Travel, Accommodations, Expenses: Amgen, Janssen

Silke Gillessen

Honoraria: Janssen

Consulting or Advisory Role: Astellas Pharma (Inst), Advanced Accelerator Applications, Roche, Janssen (Inst), Innocrin Pharma (Inst), Sanofi, Bayer (Inst) Orion Pharma GmbH, Clovis Oncology (Inst), Menarini Silicon Biosystems (Inst), Tolero Pharmaceuticals (Inst), Vaniam Group (Inst)

Patents, Royalties, Other Intellectual Property: Method for biomarker(WO 3752009138392 A1)

Other Relationship: ProteoMediX, Aranda (Inst)

\section{Yohann Loriot}

Honoraria: Sanofi, Pfizer

Consulting or Advisory Role: Janssen, Janssen (Inst), Astellas Pharma, Roche, AstraZeneca, MSD Oncology, MSD Oncology (Inst), Clovis Oncology, Seattle Genetics, Bristol-Myers Squibb

Research Funding: Sanofi (Inst), Janssen Oncology (Inst), MSD Oncology (Inst), AstraZeneca (Inst), Clovis Oncology (Inst), Exelixis (Inst), Boehringer Ingelheim (Inst), Incyte (Inst), Pfizer (Inst), Oncogenex (Inst), Medivation (Inst), CureVac (Inst), Nektar (Inst)

Travel, Accommodations, Expenses: Astellas Pharma, Janssen Oncology, Roche, MSD Oncology, AstraZeneca, Seattle Genetics

Nicolas Mottet

Honoraria: Janssen-Cilag, Astellas Pharma, Bayer, IPSEN, Sanofi/Aventis Consulting or Advisory Role: Janssen-Cilag, Astellas Pharma, Bayer, Arquer

Howard Sandler

Stock and Other Ownership Interests: Radiogel

Consulting or Advisory Role: Janssen

Other Relationship: Caribou Publishing

Daniel E. Spratt

Consulting or Advisory Role: Blue Earth Diagnostics, Janssen Oncology, AstraZeneca

Matthew R. Sydes

Honoraria: Lilly, Sanofi, Janssen, Janssen

Research Funding: Astellas Pharma, Janssen-Cilag, Pfizer, Novartis, Sanofi, Clovis Oncology 
Bertrand Tombal

Honoraria: Amgen, Astellas Pharma, Bayer, Ferring, Sanofi, Janssen, Pfizer, Myovant Sciences

Consulting or Advisory Role: Astellas Pharma, Bayer, Ferring, Janssen, Takeda, Steba Biotech, Sanofi

Speakers' Bureau: Amgen, Janssen

Research Funding: Ferring (Inst)

Travel, Accommodations, Expenses: Amgen, Astellas Pharma, Bayer, Ferring, Janssen, Sanofi

Scott Williams

Consulting or Advisory Role: Amgen, Janssen (Inst)

Travel, Accommodations, Expenses: Astellas Pharma
Christopher J. Sweeney

Stock and Other Ownership Interests: Leuchemix

Consulting or Advisory Role: Sanofi, Janssen Biotech, Astellas Pharma, Bayer,

Genentech/Roche, AstraZeneca, Pfizer, Amgen, Celgene, Lilly

Research Funding: Janssen Biotech (Inst), Astellas Pharma (Inst), Sanofi (Inst), Bayer (Inst), Dendreon, Pfizer (Inst)

Patents, Royalties, Other Intellectual Property: Leuchemix, Parthenolide,

Dimethylaminoparthenolide.Exelixis: Abiraterone plus cabozantinib combination

No other potential conflicts of interest were reported. 


\section{APPENDIX Writing Committee}

Coordinating Center at Dana-Farber Cancer Institute. Christopher J. Sweeney, Chair (Lank Center for Genitourinary Oncology, Dana-Farber Cancer Institute, Boston, MA); Meredith M. Regan (Division of Biostatistics, Dana-Farber Cancer Institute, Boston, MA); Wanling Xie (Division of Biostatistics, Dana-Farber Cancer Institute, Boston, MA).

Members Independent of Coordinating Center overseeing Statistical Analysis Plan. Marc Buyse (International Drug Development Institute, Louvain la Neuve, Belgium); Susan Halabi (Department of Biostatistics and Bioinformatics, Duke University, Durham, NC); Philip W. Kantoff (Department of Medicine, Memorial Sloan-Kettering Cancer Center, New York, NY, Prostate Cancer Foundation, Santa Monica, CA); Oliver Sartor (Departments of Medicine \& Urology, Tulane University, New Orleans, LA); Howard Soule (Prostate Cancer Foundation, Santa Monica, CA).

\section{External Review}

Donald Berry (Department of Biostatistics, The University of Texas MD Anderson Cancer Center); Noel Clarke (Urological Oncology, The Christie NHS Foundation Trust, Manchester, United Kingdom); Laurence Collette (EORTC Headquarters, Brussels, Belgium); Richard De Abreu Lourenco (University of Technology Sydney, Sydney, NSW, Australia); Mario Eisenberger (Sidney Kimmel Comprehensive Cancer Center, Johns Hopkins University, Baltimore, MD); James Dignam (Department of Public Health Science, University of Chicago, Chicago, IL); Nicholas James (University Hospitals Birmingham NHS Foundation Trust); Karim Fizazi (Department of Cancer Medicine, Institut Gustave Roussy, Villejuif, France); Silke Gillessen (Division of Cancer
Sciences, University of Manchester and The Christie, Manchester, United Kingdom, and Department of Oncology); Yohann Loriot (Department of Cancer Medicine, Institut Gustave Roussy, Villejuif, France); Nicolas Mottet (Urology Oncology, University Jean Monnet, St Etienne, France); Wendy Parulekar (Canadian Cancer Trials Group, Cancer Research Institute, Queen's University, Kinston, Ontario, Canada); Howard Sandler (Radiation Oncology, Cedars Sinai Medical Center, Los Angeles, CA); Daniel E. Spratt (University of Michigan); Matthew R. Sydes (MRC Clinical Trials Unit, Institute of Clinical Trials and Methodology, UCL, London, United Kingdom); Bertrand Tombal (Institut de Recherche Clinique, Université Catholique de Louvain, Brussels, Belgium); and Scott Williams (Radiation Oncology, Peter MacCallum Cancer Centre, Melbourne, Australia).

\section{Working Group Members: Intermediate Clinical Endpoints in Cancer of the Prostate (ICECaP) Working Group Members (in alphabetical order)}

John Armstrong, Gerhardt Attard, Donald Berry, Michel Bolla, Marc Buyse, Joseph Chin, Simon Chowdhury, Noel Clarke, Laurence Collette, Ian D. Davis, Jim Denham, Savino Mauro Di Stasi, James Dignam, Mario Eisenberger, Karim Fizazi, Boris Freidlin, Susan Halabi, Anis Hamid, Nicholas James, Philip W. Kantoff, Gary Kelloff, Malcolm Mason, Andrea Miyahira, Alicia Morgans, Nicolas Mottet, Wendy Parulekar, Meredith M. Regan, Mack Roach, Howard Sandler, Oliver Sartor, Howard Scher, Richard Simon, Jonathan Simons, Howard Soule, Srikala Sridar, Martin Stockler, Christopher J. Sweeney, Matthew R. Sydes, Bertrand Tombal, Cecile Vicier, Thomas Wiegel, Scott Williams, Manfred Wirth, Wanling Xie, Eric Yeoh, Almudena Zapatero. 